\title{
A B-spline collocation method for solving fractional diffusion and fractional diffusion-wave equations
}

\author{
A. Esen, O. Tasbozan, Y. Ucar, N.M. Yagmurlu \\ Inonu University, Faculty of Art and Sciences, Department of Mathematics, 44280 Malatya, Turkey \\ E-mail: alaattin.esen@inonu.edu.tr
}

\begin{abstract}
In this paper, we have considered the fractional diffusion and fractional diffusionwave equations in which the time derivative is a fractional derivative in the Caputo form and have obtained their numerical solutions by collocation method using cubic B-spline base functions. In the solution process, for the fractional diffusion equation $L 1$ discretizaton formula of the fractional derivative is applied, and for the fractional diffusion-wave equation $L 2$ discretizaton formula of the fractional derivative is applied. Accuracy of the proposed method is discussed by computing the error norms $L_{2}$ and $L_{\infty}$. A stability analysis of the approximation obtained by the scheme shows that the method is unconditionally stable.
\end{abstract}

2010 Mathematics Subject Classification. 80M10. 65L60, 35R11, 45A15

Keywords. Finite element method, Collocation method, Fractional diffusion equation, Fractional diffusion-wave equation, Cubic B-spline.

\section{Introduction}

The concept of differentiation and integration to non-integer order is by no means new. Interest in this subject was evident almost as soon as the ideas of the classical calculus were known [1]. However in the last few decades many authors pointed out that derivatives and integrals of non-integer order are very suitable for the description of various materials, e.g. polymers. It has been shown that new fractional-order models are more adequate than prviously used integer-order models. The growing number of fractional derivative applications in various fields of science and engineering indicates that there is a significant demand for better mathematical models of real objects, and that the fractional calculus provides one possible approach on the way to more adequate mathematical modelling of real objects and processes. For example, the modelling of diffusion in a specific type of porous medium (in fractional media) is one of the most significant applications of fractional-order derivatives [2], and the fractional diffusion-wave equations have been proposed to deal with viscoelastic problems such as propogation of stress waves in viscoelastic solids $[3,4]$. Several other examples can also be found in the literature. Although there are few analytical methods such as found by Refs. $[5,6,7,8]$ providing exact solutions of the fractional equations, the numerical methods are the most appropriate and even sometimes the only way to handle most of the problems involving fractional equations. Thus effective, accurate and easily implemented numerical methods are of great importance. Though there have been many methods applied to solve fractional partial differential equations, there is still a long way to go in this field. 
The finite element method, in particular, has been an important method for solving both ordinary and partial differential equations. But, in this paper, the finite element method is applied to solve fractional differential equations, namely fractional diffusion and fractional diffusion-wave equations. The main idea behind the finite element method is to divide the whole region of the given problem into an equivalent system of finite elements with associated nodes and to choose the most appropriate element type to model most closely the actual physical behavior . Thus, by means of the finite element method, a huge problem is converted into many solvable small problems. Those elements must be made small enough to give usable results and yet large enough to reduce computational effort [9].

There are several studies about fractional equations in the literature. For example, Sun et al. [10] have used a semi-analytical finite element method for a class of timefractional diffusion equations. Murillo and Yuste [11] have used an explicit difference for solving fractional diffusion and diffusion-wave equations in the Caputo form. Sweilam et al. [12] solved time-fractional diffusion equation by using Crank-Nicolson finite difference method. Monami and Odibat [13] have implemented relatively new analytical techniques, the variational iteration method and the Adomian decomposition method, for solving linear fractional partial differential equations arising in fluid mechanics. Çelik and Duman [14] have used Crank-Nicolson method for the fractional diffusion equation with the Riesz fractional derivative and obtained numerical results using fractional centered difference approach. A recent search of the literature shows that there are several articles recently published in the literature about the subject. Several authors have used various techniques and methods to solve this kind of problems. Among others, Tadjeran et. al. [15] have used second-order accurate numerical approximation for the fractional diffusion equation. While Lin and $\mathrm{Xu}[16]$ have applied finite difference/spectral approximations for the time-fractional diffusion equation, Daftardar-Gejji and Bhalekar [17] have solved fractional diffusion-wave equations using a new iterative method. Khader [18] has penned an article on the numerical solution for the fractional diffusion equation, and Garg and Manohar [19] have obtained numerical solution of fractional diffusion-wave equation with two space variables by matrix method. Mitkowski [20] has suggested some improvements of computational algorithms for the solution of the fractional differential equations, whereas Khader et al. [21] have proposed an efficient numerical method for solving the fractional diffusion equation. Moreover, Hanert [22] has presented a flexible numerical scheme for the discretization of the space-time fractional diffusion equation, and Heydari et al. [23] have proposed wavelets method for the time fractional diffusion-wave equation. One can find more information in those papers and other ones therein. In this paper, we will use Galerkin finite element method to obtain the numerical solutions of the diffusion and diffusion-wave equations by using the $L 1$ and $L 2$ discretizaton formula of the fractional derivative as used by Ref. [11].

The general form of the fractional diffusion and fractional diffusion-wave equations which are going to be used as a model is given by

$$
\frac{\partial^{\gamma} u}{\partial t^{\gamma}}=K \frac{\partial^{2} u}{\partial x^{2}}
$$

where 


$$
\frac{\partial^{\gamma}}{\partial t^{\gamma}} f(t)=\frac{1}{\Gamma(n-\gamma)} \int_{0}^{t}(t-\tau)^{n-\gamma-1} \frac{\partial^{n} f(\tau)}{\partial t^{n}} d \tau \quad n-1<\gamma<n
$$

is the fractional derivative in the Caputo's sense $[2,5], K$ is the diffusion coefficient and $n$ is an integer. In all numerical computations, diffusion coefficient $K$ is going to be taken as 1 . For $0<\gamma \leq 1$, Eq. (1) is the fractional diffusion equation or sub-diffusion equation, and for $1<\gamma \leq 2$ it is fractional diffusion-wave equation. In this paper, for diffusion equation, we will take the boundary conditions of the model problem (1) given in the interval $0 \leq x \leq \pi$ as

$$
u(0, t)=0, u(\pi, t)=0
$$

and the initial condition as

$$
u(x, 0)=\sin x
$$

and for diffusion-wave equation, along with the above boundary and the initial conditions, the following additional initial condition

$$
\left.\frac{\partial u(x, t)}{\partial t}\right|_{t=0}=0
$$

will be taken. The exact solution of both the problems obtained by Adomian Decomposotion Method is given as $[6]$

$$
u(x, t)=E_{\gamma}\left(-t^{\gamma}\right) \sin x
$$

where $E_{\gamma}$ is the Mittag-Leffler function [2].

In our numerical solutions, to obtain a finite element scheme for solving the fractional diffusion equation $(0<\gamma \leq 1)$, as Ref. [11] used in his explicit finite difference method, we will also discretize the Caputo derivative by means of the so-called $L 1$ formula [1]

$$
\left.\frac{\partial^{\gamma} f}{\partial t^{\gamma}}\right|_{t_{n}}=\frac{(\Delta t)^{-\gamma}}{\Gamma(2-\gamma)} \sum_{k=0}^{n-1} b_{k}^{\gamma}\left[f\left(t_{n-k}\right)-f\left(t_{n-1-k}\right)\right]+O(\Delta t)
$$

where

$$
b_{k}^{\gamma}=(k+1)^{1-\gamma}-k^{1-\gamma}
$$

and to solve the fractional diffusion-wave equation $(1<\gamma \leq 2)$, we discretize the Caputo derivative by means of the so-called $L 2$ formula [1]

$$
\left.\frac{\partial^{\gamma} f}{\partial t^{\gamma}}\right|_{t_{n}}=\frac{(\Delta t)^{-\gamma}}{\Gamma(3-\gamma)} \sum_{k=0}^{n-1} b_{k}^{\gamma}\left[f\left(t_{n-k}\right)-2 f\left(t_{n-1-k}\right)+f\left(t_{n-2-k}\right)\right]+O(\Delta t)
$$

where

$$
b_{k}^{\gamma}=(k+1)^{2-\gamma}-k^{2-\gamma} .
$$




\section{Cubic B-spline Finite Element Collocation Solutions}

Before solving Eq. (1) with the boundary conditions (3) and the initial conditions (4)-(5) by using collocation finite element method, we firstly define cubic B-spline base functions. Let us consider the interval $[a, b]$ is partitioned into $N$ finite elements of uniformly equal length by the knots $x_{m}, m=0,1,2, \ldots, N$ such that $a=x_{0}<x_{1} \cdots<x_{N}=b$ and $h=x_{m+1}-x_{m}$. The cubic B-splines $\varphi_{m}(x),(m=-1(1) N+1)$, at the knots $x_{m}$ are defined over the interval $[a, b]$ by $[24]$

$$
\varphi_{m}(x)=\frac{1}{h^{3}}\left\{\begin{array}{lc}
\left(x-x_{m-2}\right)^{3}, & x \in\left[x_{m-2}, x_{m-1}\right], \\
h^{3}+3 h^{2}\left(x-x_{m-1}\right)+3 h\left(x-x_{m-1}\right)^{2}-3\left(x-x_{m-1}\right)^{3}, & x \in\left[x_{m-1}, x_{m}\right], \\
h^{3}+3 h^{2}\left(x_{m+1}-x\right)+3 h\left(x_{m+1}-x\right)^{2}-3\left(x_{m+1}-x\right)^{3}, & x \in\left[x_{m}, x_{m+1}\right], \\
\left(x_{m+2}-x\right)^{3}, & x \in\left[x_{m+1}, x_{m+2}\right], \\
0 & \text { otherwise. }
\end{array}\right.
$$

The set of splines $\left\{\varphi_{-1}(x), \varphi_{0}(x), \ldots, \varphi_{N+1}(x)\right\}$ forms a basis for the functions defined over $[a, b]$. Therefore, an approximation solution $U_{N}(x, t)$ can be written in terms of the cubic B-splines trial functions as:

$$
U_{N}(x, t)=\sum_{m=-1}^{N+1} \delta_{m}(t) \varphi_{m}(x)
$$

where $\delta_{m}(t)$ 's are unknown, time dependent quantities to be determined from the boundary and cubic B-spline collocation conditions. Each cubic B-spline covers four elements so that each element $\left[x_{m}, x_{m+1}\right]$ is covered by four cubic B-splines. For this problem, the finite elements are identified with the interval $\left[x_{m}, x_{m+1}\right]$ and the elements knots $x_{m}, x_{m+1}$. Using the nodal values $U_{m}, U_{m}^{\prime}$ and $U_{m}^{\prime \prime}$ given in terms of the parameter $\delta_{m}(t)$

$$
\begin{aligned}
& U_{m}=U\left(x_{m}\right)=\delta_{m-1}(t)+4 \delta_{m}(t)+\delta_{m+1}(t), \\
& U_{m}^{\prime}=U^{\prime}\left(x_{m}\right)=\frac{3}{h}\left(-\delta_{m-1}(t)+\delta_{m+1}(t)\right), \\
& U_{m}^{\prime \prime}=U^{\prime \prime}\left(x_{m}\right)=\frac{6}{h^{2}}\left(\delta_{m-1}(t)-2 \delta_{m}(t)+\delta_{m+1}(t)\right),
\end{aligned}
$$

the variation of $U_{N}(x, t)$ over the typical element $\left[x_{m}, x_{m+1}\right]$ is given by

$$
U_{N}(x, t)=\sum_{j=m-1}^{m+2} \delta_{j}(t) \varphi_{j}(x) .
$$

If we substitute the global approximation (8) and its necessary derivatives (9) into Eq. (1), we obtain the following set of the first order ordinary differential equations:

$$
\dot{\delta}_{m-1}(t)+4 \dot{\delta}_{m}(t)+\dot{\delta}_{m+1}(t)-\frac{6}{h^{2}}\left(\delta_{m-1}(t)-2 \delta_{m}(t)+\delta_{m+1}(t)\right)=0
$$

where dot denotes $\gamma^{\text {th }}$ fractional derivative with respect to time. For fractional diffusion equation $(0<\gamma \leq 1)$, if time parameters $\delta_{m}(t)$ 's and its fractional time derivatives $\dot{\delta}_{m}(t)$ 's in Eq. (11) are discretized by the Crank-Nicolson formula and $L 1$ formula, respectively: 


$$
\delta=\frac{1}{2}\left(\delta^{n}+\delta^{n+1}\right)
$$

and

$$
\dot{\delta}=\frac{d^{\gamma} \delta}{d t^{\gamma}}=\frac{(\Delta t)^{-\gamma}}{\Gamma(2-\gamma)} \sum_{k=0}^{n-1}\left[(k+1)^{1-\gamma}-k^{1-\gamma}\right]\left[\delta^{n-k}-\delta^{n-k-1}\right],
$$

we obtain a recurrence relationship between successive time levels relating unknown parameters $\delta_{m}^{n+1}(t)$

$$
\begin{aligned}
& (1-\alpha) \delta_{m-1}^{n+1}+(4+2 \alpha) \delta_{m}^{n+1}+(1-\alpha) \delta_{m+1}^{n+1}=(1+\alpha) \delta_{m-1}^{n}+(4-2 \alpha) \delta_{m}^{n} \\
& +(1+\alpha) \delta_{m+1}^{n}-\sum_{k=1}^{n}\left[(k+1)^{1-\gamma}-k^{1-\gamma}\right]\left[\left(\delta_{m-1}^{n-k+1}-\delta_{m-1}^{n-k}\right)+4\left(\delta_{m}^{n-k+1}-\delta_{m}^{n-k}\right)\right. \\
& \left.+\left(\delta_{m+1}^{n-k+1}-\delta_{m+1}^{n-k}\right)\right]
\end{aligned}
$$

where

$$
\alpha=\frac{3(\Delta t)^{\gamma} \Gamma(2-\gamma)}{h^{2}}
$$

Now, for fractional diffusion-wave equation $(1<\gamma \leq 2)$, if time parameters $\delta_{m}(t)$ 's and its fractional time derivatives $\dot{\delta}_{m}(t)$ 's in Eq. (11) are discretized by the Crank-Nicolson formula and $L 2$ formula, respectively

$$
\delta=\frac{1}{2}\left(\delta^{n}+\delta^{n+1}\right)
$$

and

$$
\dot{\delta}=\frac{d^{\gamma} \delta}{d t^{\gamma}}=\frac{(\Delta t)^{-\gamma}}{\Gamma(3-\gamma)} \sum_{k=0}^{n-1}\left[(k+1)^{2-\gamma}-k^{2-\gamma}\right]\left[\delta^{n-k}-2 \delta^{n-k-1}+\delta^{n-k-2}\right],
$$

we obtain a recurrence relationship between successive time levels relating unknown parameters $\delta_{m}^{n+1}(t)$

$$
\begin{aligned}
& (1-\alpha) \delta_{m-1}^{n+1}+(4+2 \alpha) \delta_{m}^{n+1}+(1-\alpha) \delta_{m+1}^{n+1}=(2+\alpha) \delta_{m-1}^{n}+(8-2 \alpha) \delta_{m}^{n}+(2+\alpha) \delta_{m+1}^{n} \\
& -\left(\delta_{m-1}^{n-1}+4 \delta_{m}^{n-1}+\delta_{m+1}^{n-1}\right)-\sum_{k=1}^{n}\left[(k+1)^{2-\gamma}-k^{2-\gamma}\right]\left[\left(\delta_{m-1}^{n-k+1}-2 \delta_{m-1}^{n-k}+\delta_{m-1}^{n-k-1}\right)\right. \\
& \left.+4\left(\delta_{m}^{n-k+1}-2 \delta_{m}^{n-k}+\delta_{m}^{n-k-1}\right)+\left(\delta_{m+1}^{n-k+1}-2 \delta_{m+1}^{n-k}+\delta_{m+1}^{n-k-1}\right)\right]
\end{aligned}
$$

where

$$
\alpha=\frac{3(\Delta t)^{\gamma} \Gamma(3-\gamma)}{h^{2}}
$$

Both the systems (13) and (15) consist of $N+1$ linear equations including $N+3$ unknown parameters $\left(\delta_{-1}, \ldots, \delta_{N+1}\right)^{T}$. To obtain a unique solution to these systems, we need two additional constraints. These are obtained from the boundary conditions and can be used to eliminate $\delta_{-1}$ and $\delta_{N+1}$ from the systems. 


\subsection{Initial state}

The initial vector $\mathbf{d}^{0}=\left(\delta_{0}, \delta_{1}, \delta_{2}, \ldots, \delta_{N-2}, \delta_{N-1}, \delta_{N}\right)^{T}$ is determined from the initial and boundary conditions. So the approximation (8) can be rewritten for the initial condition as

$$
U_{N}(x, 0)=\sum_{m=-1}^{N+1} \delta_{m}(0) \varphi_{m}(x)
$$

where the $\delta_{m}(0)$ 's are unknown parameters. We require the initial numerical approximation $U_{N}(x, 0)$ satisfy the following conditions:

$$
\begin{aligned}
& U_{N}(x, 0)=U\left(x_{m}, 0\right), \quad m=0,1, \ldots, N \\
& \left(U_{N}\right)_{x x}(0,0)=0, \quad\left(U_{N}\right)_{x x}(\pi, 0)=0 .
\end{aligned}
$$

Thus, using the these conditions leads to a three-diagonal system of matrix of the form

$$
W \mathbf{d}^{0}=\mathbf{b}
$$

where

$$
W=\left[\begin{array}{llllllll}
6 & 0 & & & & & & \\
1 & 4 & 1 & & & & & \\
& 1 & 4 & 1 & & & & \\
& & & & \ddots & & & \\
& & & & & 1 & 4 & 1 \\
& & & & & & 0 & 6
\end{array}\right]
$$

and

$$
\mathbf{b}=\left(U\left(x_{0}, 0\right), U\left(x_{1}, 0\right), U\left(x_{2}, 0\right), \ldots, U\left(x_{N-2}, 0\right), U\left(x_{N-1}, 0\right), U\left(x_{N}, 0\right)\right)^{T} .
$$

The obtained numerical results are compared with other published ones available in the literature.

\subsection{Stability analysis}

The investigation of the stability of the approximation obtained by the algorithm will be based on the von Neumann stability analysis in which the growth factor of a typical Fourier mode is defined as:

$$
\delta_{m}^{n}=\xi^{n} e^{i m \varphi}
$$

where $i=\sqrt{-1}$. Firstly, substituting the Fourier mode (19) into the recurrence relationship (13), we obtain

$$
\begin{aligned}
& \xi^{n+1}\left((1-\alpha) e^{-i \varphi}+(4+2 \alpha)+(1-\alpha) e^{i \varphi}\right)=\xi^{n}\left((1+\alpha) e^{-i \varphi}+(4-2 \alpha)+(1+\alpha) e^{i \varphi}\right) \\
& -\sum_{k=1}^{n}\left[(k+1)^{1-\gamma}-k^{1-\gamma}\right]\left[\left(\xi^{n-k+1}-\xi^{n-k}\right)\left(e^{-i \varphi}+4+e^{i \varphi}\right)\right] .
\end{aligned}
$$

Next, if we write 


$$
\xi^{n+1}=\zeta \xi^{n}
$$

and assume that

$$
\zeta \equiv \zeta(\varphi)
$$

is independent of time, then we get the following expression for the amplification factor $\zeta$ of the sub-diffusion mode:

$$
\begin{aligned}
& \zeta\left((1-\alpha) e^{-i \varphi}+(4+2 \alpha)+(1-\alpha) e^{i \varphi}\right)=\left((1+\alpha) e^{-i \varphi}+(4-2 \alpha)+(1+\alpha) e^{i \varphi}\right) \\
& -\sum_{k=1}^{n}\left[(k+1)^{1-\gamma}-k^{1-\gamma}\right]\left[\left(\zeta^{n-k+1}-\zeta^{n-k}\right)\left(e^{-i \varphi}+4+e^{i \varphi}\right)\right] .
\end{aligned}
$$

For the given scheme to be stable in terms of Fourier stability analysis, the condition $|\zeta| \leq 1$ must be satisfied. Considering the extreme value $\zeta=1$, from the Eqs. (20) and (22), we obtain the following inequality

$$
8 \alpha \sin ^{2}(\varphi / 2) \geq 0 \text {. }
$$

Substituting the Fourier mode (19) into the recurrence relationship (15), and following similar processes, we again obtain(23) .Since $\alpha \geq 0$, both of the schemes are unconditionally stable.

\section{$3 \quad$ Numerical examples and results}

Numerical results for the diffusion and diffusion-wave problems are obtained by collocation method using cubic B-spline base functions. The accuracy of the method is measured by the error norm $L_{2}$

$$
L_{2}=\left\|U^{\text {exact }}-U_{N}\right\|_{2} \simeq \sqrt{h \sum_{j=0}^{N}\left|U_{j}^{\text {exact }}-\left(U_{N}\right)_{j}\right|^{2}}
$$

and the error norm $L_{\infty}$

$$
L_{\infty}=\left\|U^{\text {exact }}-U_{N}\right\|_{\infty} \simeq \max _{j}\left|U_{j}^{\text {exact }}-\left(U_{N}\right)_{j}\right| .
$$

The comparison of the analytical solution and numerical solutions obtained for diffusion equation for values of $\gamma=0.25, \gamma=0.50$ and $\gamma=0.75$ is given in Table 1 . As it is clearly seen from the table, the analytical and numerical solutions obtained by the present scheme are in good agreement with each other. As the value of $\gamma$ increases, the values of error norms $L_{2}$ and $L_{\infty}$ decrease. In Table 2, we demonstrate the numerical results for $\gamma=0.5$, $\Delta t=0.0007$ and $t_{f}=0.35$ and for different number of divisions of the region. Table 2 clearly shows that as the number of division increases, the obtained numerical results become more accurate. We see this from the decreasing values of the error norms $L_{2}$ and $L_{\infty}$. In Figure 1, we demonstrate the graphs of numerical solutions obtained for $\gamma=0.50$ and $N=40$ at different time levels. In Table 3, we demonstrate the error norms $L_{2}$ and $L_{\infty}$ for $\gamma=0.25, \gamma=0.50$ and $\gamma=0.75$ at various time levels. 
TABLE 1. The comparison of the exact solutions with the numerical solutions of the diffusion problem with $N=40, \Delta t=0.0007$ and $t_{f}=0.35$ for different values of $\gamma$ and the error norms $L_{2}$ and $L_{\infty}$.

\begin{tabular}{|c|c|c|c|c|c|c|}
\hline \multirow{2}{*}{$x$} & \multicolumn{2}{|c|}{$\gamma=0.25$} & \multicolumn{2}{|c|}{$\gamma=0.50$} & \multicolumn{2}{|c|}{$\gamma=0.75$} \\
\hline & Numerical & Exact & Numerical & Exact & Numerical & Exact \\
\hline 0.000000 & 0.000000 & 0.000000 & 0.000000 & 0.000000 & 0.000000 & 0.000000 \\
\hline 0.314159 & 0.164069 & 0.164109 & 0.176594 & 0.176627 & 0.194605 & 0.194621 \\
\hline 0.628319 & 0.312079 & 0.312153 & 0.335901 & 0.335965 & 0.370160 & 0.370192 \\
\hline 0.942478 & 0.429539 & 0.429642 & 0.462328 & 0.462416 & 0.509482 & 0.509525 \\
\hline 1.256637 & 0.504954 & 0.505074 & 0.543499 & 0.543602 & 0.598932 & 0.598983 \\
\hline 1.570796 & 0.530940 & 0.531066 & 0.571469 & 0.571577 & 0.629754 & 0.629808 \\
\hline 1.884956 & 0.504954 & 0.505074 & 0.543499 & 0.543602 & 0.598932 & 0.598983 \\
\hline 2.199115 & 0.429539 & 0.429642 & 0.462328 & 0.462416 & 0.509482 & 0.509525 \\
\hline 2.513274 & 0.312079 & 0.312153 & 0.335901 & 0.335965 & 0.370160 & 0.370192 \\
\hline 2.827433 & 0.164069 & 0.164109 & 0.176594 & 0.176627 & 0.194605 & 0.194621 \\
\hline 3.141593 & 0.000000 & 0.000000 & 0.000000 & 0.000000 & 0.000000 & 0.000000 \\
\hline$L_{2} \times 10^{3}$ & 0.158757 & & 0.135759 & & 0.066844 & \\
\hline$L_{\infty} \times 10^{3}$ & 0.126670 & & 0.108320 & & 0.053334 & \\
\hline
\end{tabular}

TABLE 2. The comparison of the exact solutions with the numerical solutions of the diffusion problem with $\gamma=0.5, \Delta t=0.0007$ and $t_{f}=0.35$ for different values of $N$ and the error norms $L_{2}$ and $L_{\infty}$.

\begin{tabular}{clllll}
\hline$x$ & $\mathrm{~N}=10$ & $\mathrm{~N}=20$ & $\mathrm{~N}=40$ & $\mathrm{~N}=80$ & Exact \\
\hline 0.000000 & 0.000000 & 0.000000 & 0.000000 & 0.000000 & 0.000000 \\
0.314159 & 0.175956 & 0.176466 & 0.176594 & 0.176625 & 0.176627 \\
0.628319 & 0.334689 & 0.335659 & 0.335901 & 0.335962 & 0.335965 \\
0.942478 & 0.460659 & 0.461994 & 0.462328 & 0.462412 & 0.462416 \\
1.256637 & 0.541537 & 0.543107 & 0.543499 & 0.543597 & 0.543602 \\
1.570796 & 0.569406 & 0.571056 & 0.571469 & 0.571572 & 0.571577 \\
1.884956 & 0.541537 & 0.543107 & 0.543499 & 0.543597 & 0.543602 \\
2.199115 & 0.460659 & 0.461994 & 0.462328 & 0.462412 & 0.462416 \\
2.513274 & 0.334689 & 0.335659 & 0.335901 & 0.335962 & 0.335965 \\
2.827433 & 0.175956 & 0.176466 & 0.176594 & 0.176625 & 0.176627 \\
3.141593 & 0.000000 & 0.000000 & 0.000000 & 0.000000 & 0.000000 \\
\hline$L_{2} \times 10^{3}$ & 2.721007 & 0.652825 & 0.135759 & 0.006493 & \\
$L_{\infty} \times 10^{3}$ & 2.171049 & 0.520879 & 0.108320 & 0.005180 & \\
\hline
\end{tabular}




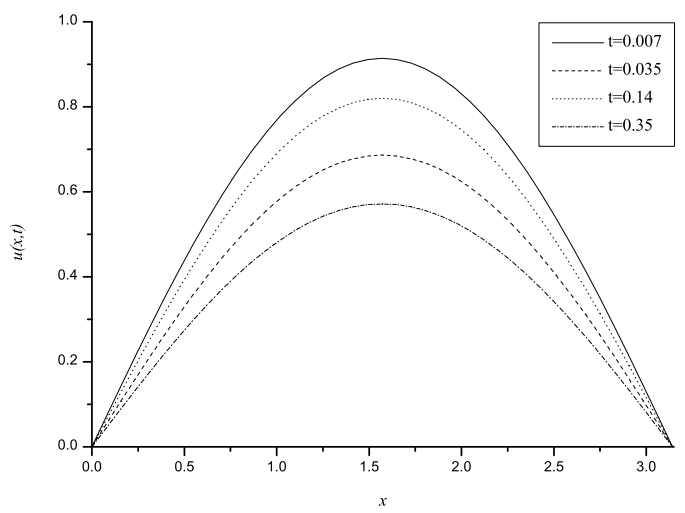

FiguRE 1. Numerical solutions of the diffusion problem for $\gamma=0.50$ and $N=40$ at different time levels.

TABLE 3. The comparison of the exact solutions with the numerical solutions of the diffusion problem with $N=40, \Delta t=0.0007$ and $t_{f}=0.35$ for different values of $N$ and the error norms $L_{2}$ and $L_{\infty}$.

\begin{tabular}{|c|c|c|c|c|c|c|}
\hline \multirow{2}{*}{$t$} & \multicolumn{2}{|c|}{$\gamma=0.25$} & \multicolumn{2}{|c|}{$\gamma=0.50$} & \multicolumn{2}{|c|}{$\gamma=0.75$} \\
\hline & $L_{2} \times 10^{3}$ & $L_{\infty} \times 10^{3}$ & $L_{2} \times 10^{3}$ & $L_{\infty} \times 10^{3}$ & $L_{2} \times 10^{3}$ & $L_{\infty} \times 10^{3}$ \\
\hline 0.07 & 0.047699 & 0.038058 & 0.240770 & 0.192106 & 0.332833 & 0.265563 \\
\hline 0.14 & 0.122036 & 0.097370 & 0.018097 & 0.014440 & 0.155756 & 0.124276 \\
\hline 0.21 & 0.143881 & 0.114801 & 0.066579 & 0.053122 & 0.052431 & 0.041834 \\
\hline 0.28 & 0.153593 & 0.122549 & 0.110137 & 0.087877 & 0.017091 & 0.013637 \\
\hline 0.35 & 0.158757 & 0.126670 & 0.135759 & 0.108320 & 0.066844 & 0.053334 \\
\hline
\end{tabular}

The comparison of the analytical solution and numerical solutions obtained by the scheme for diffusion-wave equation for values of $\gamma=1.25, \gamma=1.50$ and $\gamma=1.75$ is given in Table 4 . The table clearly demonstrate that the obtained numerical results are satisfactorily in good agreement with analytical ones. As the value $\gamma$ increases, so the values of the error norms $L_{2}$ and $L_{\infty}$. In Table 5 , the numerical results for $\gamma=1.5$, $\Delta t=0.0075$ and $t_{f}=3.75$ for various values of $N$ are given. As it is seen from the table, as the number of division increases, the values of error decrease. In Figure 2, the numerical solutions for the values of $\gamma=1.50$ and $N=40$ at various time levels are presented. In Table 6 , we show the error norms $L_{2}$ and $L_{\infty}$ for $N=40, \Delta t=0.0075$ and $t_{f}=3.75$ at different values of $\gamma$ and $t$. 
TABLE 4. The comparison of the exact solutions with the numerical solutions of the diffusion-wave problem with $N=40, \Delta t=0.0075$ and $t_{f}=3.75$ for different values of $\gamma$ and the error norms $L_{2}$ and $L_{\infty}$.

\begin{tabular}{|c|c|c|c|c|c|c|}
\hline \multirow{2}{*}{$x$} & \multicolumn{2}{|c|}{$\gamma=1.25$} & \multicolumn{2}{|c|}{$\gamma=1.50$} & \multicolumn{2}{|c|}{$\gamma=1.75$} \\
\hline & Numerical & Exact & Numerical & Exact & Numerical & Exact \\
\hline 0.000000 & 0.000000 & 0.000000 & 0.000000 & 0.000000 & 0.000000 & 0.000000 \\
\hline 0.314159 & -0.030443 & -0.030452 & -0.073430 & -0.073478 & -0.137708 & -0.137943 \\
\hline 0.628319 & -0.057907 & -0.057923 & -0.139672 & -0.139763 & -0.261937 & -0.262384 \\
\hline 0.942478 & -0.079702 & -0.079724 & -0.192242 & -0.192367 & -0.360525 & -0.361140 \\
\hline 1.256637 & -0.093695 & -0.093721 & -0.225993 & -0.226141 & -0.423823 & -0.424546 \\
\hline 1.570796 & -0.098517 & -0.098545 & -0.237624 & -0.237779 & -0.445634 & -0.446394 \\
\hline 1.884956 & -0.093695 & -0.093721 & -0.225993 & -0.226141 & -0.423823 & -0.424546 \\
\hline 2.199115 & -0.079702 & -0.079724 & -0.192242 & -0.192367 & -0.360525 & -0.361140 \\
\hline 2.513274 & -0.057907 & -0.057923 & -0.139672 & -0.139763 & -0.261937 & -0.262384 \\
\hline 2.827433 & -0.030443 & -0.030452 & -0.073430 & -0.073478 & -0.137708 & -0.137943 \\
\hline 3.141593 & 0.000000 & 0.000000 & 0.000000 & 0.000000 & 0.000000 & 0.000000 \\
\hline$L_{2} \times 10^{3}$ & 0.035040 & & 0.194207 & & 0.952239 & \\
\hline$L_{\infty} \times 10^{3}$ & 0.027958 & & 0.154955 & & 0.759777 & \\
\hline
\end{tabular}

TABLE 5. The comparison of the exact solutions with the numerical solutions of the diffusion-wave problem with $\gamma=1.5, \Delta t=0.0075$ and $t_{f}=3.75$ for different values of $N$ and the error norms $L_{2}$ and $L_{\infty}$.

\begin{tabular}{crrrrr}
\hline$x$ & $\mathrm{~N}=10$ & $\mathrm{~N}=20$ & $\mathrm{~N}=40$ & $\mathrm{~N}=80$ & \multicolumn{1}{c}{ Exact } \\
\hline 0.000000 & 0.000000 & 0.000000 & 0.000000 & 0.000000 & 0.000000 \\
0.314159 & -0.072637 & -0.073272 & -0.073430 & -0.073469 & -0.073478 \\
0.628319 & -0.138164 & -0.139372 & -0.139672 & -0.139746 & -0.139763 \\
0.942478 & -0.190167 & -0.191829 & -0.192242 & -0.192345 & -0.192367 \\
1.256637 & -0.223554 & -0.225508 & -0.225993 & -0.226115 & -0.226141 \\
1.570796 & -0.235059 & -0.237114 & -0.237624 & -0.237751 & -0.237779 \\
1.884956 & -0.223554 & -0.225508 & -0.225993 & -0.226115 & -0.226141 \\
2.199115 & -0.190167 & -0.191829 & -0.192242 & -0.192345 & -0.192367 \\
2.513274 & -0.138164 & -0.139372 & -0.139672 & -0.139746 & -0.139763 \\
2.827433 & -0.072637 & -0.073272 & -0.073430 & -0.073469 & -0.073478 \\
3.141593 & 0.000000 & 0.000000 & 0.000000 & 0.000000 & 0.000000 \\
\hline$L_{2} \times 10^{3}$ & 3.408666 & 0.833398 & 0.194207 & 0.034703 & \\
$L_{\infty} \times 10^{3}$ & 2.719722 & 0.664955 & 0.154955 & 0.027689 & \\
\hline
\end{tabular}




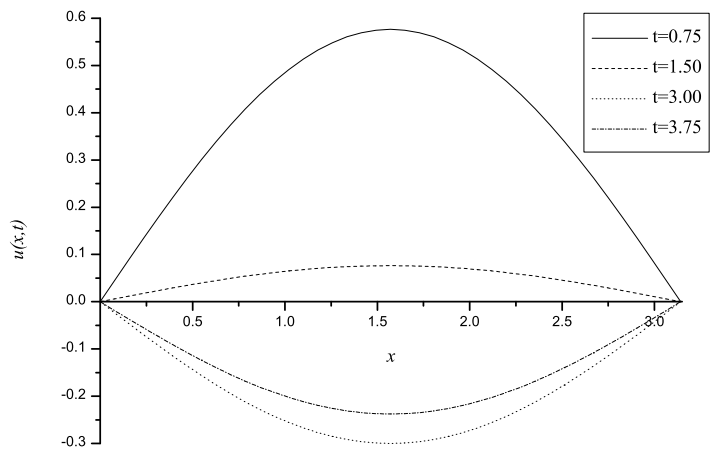

FiguRE 2. Numerical solutions of the diffusion-wave problem for $\gamma=1.50$ and $N=40$ at different time levels.

TABLE 6. The comparison of the exact solutions with the numerical solutions of the diffusion problem with $N=40, \Delta t=0.0075$ and $t_{f}=3.75$ for different values of $N$ and the error norms $L_{2}$ and $L_{\infty}$.

\begin{tabular}{|c|c|c|c|c|c|c|}
\hline \multirow[b]{2}{*}{$t$} & \multicolumn{2}{|c|}{$\gamma=1.25$} & \multicolumn{2}{|c|}{$\gamma=1.50$} & \multicolumn{2}{|c|}{$\gamma=1.75$} \\
\hline & $L_{2} \times 10^{3}$ & $L_{\infty} \times 10^{3}$ & $L_{2} \times 10^{3}$ & $L_{\infty} \times 10^{3}$ & $L_{2} \times 10^{3}$ & $L_{\infty} \times 10^{3}$ \\
\hline 1.5 & 0.264233 & 0.210828 & 0.271215 & 0.216398 & 0.416904 & 0.332641 \\
\hline 3.0 & 0.045250 & 0.036104 & 0.065312 & 0.052112 & 0.954343 & 0.761455 \\
\hline 4.5 & 0.068853 & 0.054936 & 0.219035 & 0.174765 & 0.403232 & 0.321732 \\
\hline 6.0 & 0.056669 & 0.045215 & 0.070316 & 0.056104 & 0.804592 & 0.641971 \\
\hline 7.5 & 0.026110 & 0.020833 & 0.045078 & 0.035967 & 0.395893 & 0.315877 \\
\hline
\end{tabular}




\section{Conclusion}

In the present study, a collocation finite element method has been successfully used to obtain the numerical solutions of diffusion and diffusion-wave equations in which the fractional derivative is of the Caputo form. In this study, the fractional derivative appearing in the fractional diffusion and diffusion-wave equations is approximated ,respectively, by means of the so-called $L 1$ and $L 2$ formulae the same as used by Ref. [11] in the explicit difference method solution. It is clearly seen from the obtained results that the employed method is a remarkably good one to obtain numerical solutions of this kind fractional partial differential equations.

\section{References}

[1] K. B. Oldham and J. Spanier, The Fractional Calculus, Academic, New York, 1974.

[2] I. Podlubny, Fractional Differential Equations, Academic Press, San Diego, 1999.

[3] F. Mainardi, Fractional Diffusive Waves in Viscoeslactic Solids., Non-linear Waves in Solids, J.L. Wegner and F.R. Norwood, eds., ASME/AMR, Fairfield, NJ, pp. 93-97, 1995.

[4] F. Mainardi and P.Paradisi, A Model of Diffusive Waves in Viscoelasticity Based on Fractional Calculus, Proceedings of the 36th Conference on Decision and Control, O.R. Gonzales, ed., San Diego, CA, pp. 4961-4966, 1997.

[5] A. A. Kilbas, H.M. Srivastava and J.J. Trujillo, Theory and Applications of Fractional Differential Equations, Elsevier, Amsterdam, 2006.

[6] S. S. Ray, Exact Solutions for Time-Fractional Diffusion-Wave Equations by Decomposition Method, Phys. Scr., 75, 53-61, 2007.

[7] O.P. Agrawal, Solution for a fractional diffusion-wave equation defined in a bounded domain, Nonlin. Dynam., 29 (2002) 145-155.

[8] H. Jafari and S.Momani, Solving fractional diffusion and waves equations by modifiying homotopy perturbation method, Phys. Lett. A, 370 (2007) 388-396.

[9] D.L. Logan, A First Course in the Finite Element Method (Fourth Edition), Thomson, 2007.

[10] H.G. Sun, W. Chen and K.Y. Sze, A semi-analytical finite element method for a class of time-fractional diffusion equations, arXiv: 1109.0641v1, [math-ph], 3 Sep 2011.

[11] J. Quintana-Murillo and S.B. Yuste, An Explicit Difference Method for solving Fractional Diffusion and Diffusion-Wave Equations in the Caputo Form, J. Comput. Nonlinear Dynam. 6, (2011) 021014. 
[12] N.H. Sweilam, M.M. Khader and A.M.S. Mahdy, Crank-Nicolson Finite Difference Method For Solving Time-Fractional Diffusion Equation, J. Fractional Calculus and Applications, 2 (2012)1-9.

[13] S. Monami and Z. Odibat, Analytical approach to linear fractional partial differential equations arising in fluid mechanics, Phys. Lett. A, 355(2006) 271-279.

[14] C. Çelik and M. Duman, Crank-Nicolson method for the fractional diffusion equation with the Riesz fractional derivative, J. Comput. Phys., 231(2012) 1743-1750.

[15] C. Tadjeran, M.M. Meerschaert and H. Scheffler, A second-order accurate numerical approximation for the fractional diffusion equation, Journal of Computational Physics, 213 (2006) 205-213.

[16] Y. Lin and C. Xu, Finite difference/spectral approximations for the time-fractional diffusion equation, Journal of Computational Physics, 225 (2007) 1533-1552.

[17] V. Daftardar-Gejji and S. Bhalekar, Solving Fractional Diffusion-Wave Equations Using a New Iterative Method, Fractional Calculus and Applied Analysis, 2(2008) 193-202.

[18] M.M. Khader, On the numerical solutions for the fractional diffusion equation, Communications in Nonlinear Science and Numerical Simulation, doi: 10.1016/j.cnsns.2010.09.007

[19] M. Garg and P. Manohar, Numerical Solution of Fractional Diffusion-wave Equation with Two Space Variables by Matrix Method, Fractional Calculus and Applied Analaysis, 2 (2010) 191-207.

[20] W. Mitkowski, Approximation of Fractional Diffusion-Wave Equation, Acta Mechanica et Automatica, 5 (2011) 65-68.

[21] M.M. Khader, N.H. Sweliam and A.M.S. Mahdy, An efficient Numerical Method for Solving the Fractional Diffusion Equation, Journal of Applied Mathematics and Bioinformatics, 2 (2011) 1-12.

[22] E. Hanert, On the numerical solution of space-time fractional diffusion models, Computers and Fluids, 46 (2011) 33-39.

[23] M.H. Heydari, M.R. Hoosmandasi, F.M. Maalek Ghaini and C. Cattani, Wavelets method for the time fractional diffusion-wave equation, Physics Letters A 379 (2015) 71-76.

[24] P. M. Prenter, Splines and Variasyonel Methods, New York, John Wiley, 1975. 